\title{
Water Vapor Profiling Using a Widely Tunable, Amplified Diode-Laser-Based Differential Absorption Lidar (DIAL)
}

\author{
AMIN R. NEHRIR AND KEVIN S. REPASKY \\ Department of Electrical and Computer Engineering, Montana State University, Bozeman, Montana \\ JOHN L. CARLSTEN \\ Department of Physics, Montana State University, Bozeman, Montana \\ MichaEl D. OBLAND \\ Department of Physics, Montana State University, Bozeman, Montana, and SSAI/NASA Langley Research Center, Hampton, Virginia
}

JOSEPH A. SHAW

Department of Electrical and Computer Engineering, Montana State University, Bozeman, Montana

(Manuscript received 28 July 2008, in final form 5 November 2008)

\begin{abstract}
A differential absorption lidar (DIAL) instrument for automated profiling of water vapor in the lower troposphere has been designed, tested, and is in routine operation at Montana State University. The laser transmitter for the DIAL instrument uses a widely tunable external cavity diode laser (ECDL) to injection seed two cascaded semiconductor optical amplifiers (SOAs) to produce a laser transmitter that accesses the 824-841-nm spectral range. The DIAL receiver utilizes a 28-cm-diameter Schmidt-Cassegrain telescope; an avalanche photodiode (APD) detector; and a narrowband optical filter to collect, discriminate, and measure the scattered light. A technique of correcting for the wavelength-dependent incident angle upon the narrowband optical filter as a function of range has been developed to allow accurate water vapor profiles to be measured down to $225 \mathrm{~m}$ above the surface. Data comparisons using the DIAL instrument and collocated radiosonde measurements are presented demonstrating the capabilities of the DIAL instrument.
\end{abstract}

\section{Introduction}

Water vapor in the lower troposphere plays an important role in many earth system processes associated with the radiation budget and climate, moisture transport and the hydrologic cycle, and weather (Trenberth et al. 2007; Dabberdt and Schlatter 1996). Water vapor is primarily contained within the lowest $3 \mathrm{~km}$ of the troposphere with high temporal and spatial fluxes (Dabberdt and Schlatter 1996; Weckwerth et al. 1999). Radiosonde launches are currently used to obtain water vapor profiles, but this measurement technique can only provide information at one location at one point in time and cannot

Corresponding author address: Kevin S. Repasky, Dept. of Electrical and Computer Engineering, Montana State University, Cobleigh Hall, Room 610, Bozeman, MT 59717.

E-mail: repasky@ece.montana.edu easily be used to monitor the spatial and temporal changes associated with atmospheric water vapor (Turner et al. 2000).

Recognizing the importance of accurate measurements of water vapor profiles in the lower atmosphere for understanding, modeling, and predicting both climate and weather, research in developing instruments for the retrieval of water vapor profiles is ongoing (Rall et al. 1996; Goldsmith et al. 1998; Wulfmeyer 1998; Wulfmeyer and Bosenberg 1998; Bosenberg 1998; Wulfmeyer and Walther 2001; Turner et al. 2002; Ehret et al. 1993; Behrendt et al. 2007; Browell et al. 1998; Ismail et al. 2000; Machol et al. 2004; Obland 2007; Obland et al. 2008, manuscript submitted to J. Appl. Remote Sens., hereafter ORNCS). Passive remote sensing instruments such as the Atmospheric Emitted Radiance Interferometer (AERI; Feltz et al. 2003) and the Atmospheric Infrared Sounder (AIRS; Divakarla et al. 
2006) show great utility for monitoring atmospheric water vapor, but measure low spatial resolution or column-integrated water vapor amounts. Active remote sensing techniques such as light detection and ranging (lidar) are capable of producing range-resolved measurements of atmospheric water vapor. Raman lidar instruments (Turner et al. 2000; Goldsmith et al. 1998; Turner et al. 2002) are capable of making range-resolved measurements of atmospheric water vapor using high-power pulsed lasers. The range information is determined by the time of flight of the light, while the molecular species information is determined by the characteristic Raman shift to longer wavelengths associated with the inelastic Raman scattering from each molecule. Raman lidar instruments typically have high peak powers due to the small Raman scattering cross sections and, furthermore, Raman lasers need to be accurately calibrated for range-resolved water vapor retrievals (Wulfmeyer and Bosenberg 1998). Differential absorption lidar (DIAL) is a second lidar method capable of obtaining range-resolved profiles of atmospheric molecular densities (Rall et al. 1996; Wulfmeyer 1998; Wulfmeyer and Bosenberg 1998; Bosenberg 1998; Ehret et al. 1993; Behrendt et al. 2007; Browell et al. 1998; Ismail et al. 2000; Machol et al. 2004; Obland 2007; ORNCS). DIAL instruments use a pulsed tunable laser transmitter that can be tuned to an online wavelength associated with an absorption feature for the molecule of interest and then tuned to an offline wavelength with no molecular absorption, or significantly less absorption in the case of a continuum, like ozone. The wavelengths of the DIAL transmitter are chosen so that the ratio of the return signal from the online and offline wavelengths is directly related to the absorption from the molecules of interest. Because the DIAL transmitter uses a ratio of the return signal from the closely spaced online and offline wavelengths, the DIAL instrument avoids the difficult calibration faced by Raman lidar instruments.

The high spatial and temporal fluxes of water vapor are hard to capture from a single location. The deployment of an array of low-cost, eye-safe, automated DIAL instruments for water vapor profiles could be useful for measuring water vapor transport, which is important for better forecasting of precipitation and understanding long-term trends needed for climate studies (Dabberdt and Schlatter 1996). Many water vapor lidar instruments currently operating are large, complex, expensive, and are not automated (Goldsmith et al. 1998; Wulfmeyer 1998; Ismail et al. 2000). However, in recent years, work has begun on developing smaller automated water vapor lidar instruments (Rall et al. 1996; Machol et al. 2004; Obland 2007; ORNCS). One promising avenue of research toward the development of DIAL instruments for water vapor studies is to use semiconductor laser transmitters. Diode lasers are compact, inexpensive, can be tuned, and have good spectral coverage in the nearinfrared spectral region where water vapor has many absorption lines. Several numerical studies of diodelaser-based transmitters and photon-counting avalanchephotodiode-based receivers have been performed (Reagan et al. 1993; Reagan et al. 1996), but few systems have been built. Rall et al. (1996) built a DIAL instrument based on a diode laser near $811.6 \mathrm{~nm}$, while Machol et al. (2004) reported initial water vapor DIAL measurements using a laser transmitter based on a distributed feedback (DFB) laser diode used to injection seed a flared amplifier. Comparisons between their DIAL retrieval and radiosonde data showed agreement up to a height of $2.5 \mathrm{~km}$. Machol et al. (2004) noted that new laser transmitter designs are needed for better spectral coverage and larger tuning ranges, calling for "a new laser with a tuning range that accesses a larger selection of good water vapor lines...." Researchers at Montana State University developed a laser transmitter for a water vapor DIAL based on a widely tunable external cavity diode laser (ECDL) that can access many water vapor lines (Repasky et al. 2004; Obland et al. 2005-2007; Nehrir et al. 2008). In his thesis work, Obland used this laser transmitter to demonstrate initial water vapor profiles up to approximately $1 \mathrm{~km}$ (Obland 2007; ORNCS). Further improvements to this water vapor DIAL instrument and water vapor profile retrieval based on the DIAL equation allow the low-power diode-laser-based DIAL instrument to accurately retrieve water vapor profiles in the lower troposphere from $225 \mathrm{~m}$ to $\sim 3.0 \mathrm{~km}$, allowing for routine nighttime measurements.

The compact diode-laser-based DIAL instrument for water vapor profiling constructed and tested at Montana State University is described in this paper. The laser transmitter is based on a tunable ECDL with a tuning range from 824 to $841 \mathrm{~nm}$. This ECDL is used to injection seed two cascaded semiconductor optical amplifiers (SOAs). The output from the second SOA is pulsed using the first-order beam from an acousto-optic modulator. Light is collected using a commercial telescope and is monitored using an avalanche photodiode (APD). Water vapor profiles collected using this DIAL instrument demonstrate good agreement with data collected using collocated radiosonde measurements.

This paper is organized as follows. The theory used to retrieve the water vapor profile is presented in section 2 . In this section, the DIAL equation is developed. The selection of the water vapor absorption line is also described in this section. The DIAL instrument and data collection are described in section 3 . The wavelength and range-dependent transmission of the narrowband optical 
filter used in the DIAL receiver and its implications on the water vapor retrieval are discussed in section 4 . Measurements of water vapor profiles made using the DIAL instrument are presented in section 5. Finally, some brief concluding remarks are presented in section 6 .

\section{Theory}

\section{a. DIAL equation}

The optical power, $P(\lambda, r)$, collected at wavelength $\lambda$ from a range of $r$ can be calculated using the lidar equation (Kovalev and Eichinger 2004):

$$
\begin{aligned}
P(\lambda, r)= & P_{t}(\lambda) \frac{A}{r^{2}} \Delta r \beta(\lambda, r) T_{A}^{2}(\lambda, r) \varepsilon_{0}(\lambda, r) \varepsilon_{R}(\lambda, r) T_{F}(\lambda, r) \\
& \times \varepsilon_{D}(\lambda, r),
\end{aligned}
$$

where $P_{t}(\lambda)$ is the transmitted optical power, $A$ is the area of the receiver, $\Delta r=\tau c / 2$ is the range bin size, $\tau$ is the pulse duration, $c$ is the speed of light, $\beta(\lambda, r)$ is the atmospheric backscatter coefficient, $T_{A}^{2}(\lambda, r)$ is the round-trip atmospheric transmission, $\varepsilon_{0}(\lambda, r)$ is the receiver overlap factor, $\varepsilon_{R}(\lambda, r)$ is the receiver optics transmission except for the narrowband optical filter, $T_{F}(\lambda, r)$ is the transmission of the narrowband optical filter, and $\varepsilon_{D}(\lambda, r)$ is the detector efficiency. The roundtrip atmospheric transmission factor can be written as

$$
T_{A}^{2}(\lambda, r)=e^{-2 \int_{0}^{r} k\left(\lambda, r^{\prime}\right) d r^{\prime}} e^{-2 \int_{0}^{r} \sigma\left(\lambda, r^{\prime}\right) N\left(r^{\prime}\right) d r^{\prime}},
$$

where $k\left(\lambda, r^{\prime}\right)$ is the atmospheric extinction factor, $\sigma\left(\lambda, r^{\prime}\right)$ is the absorption cross section for the molecule of interest, and $N\left(r^{\prime}\right)$ is the number density of the absorbing molecule of interest.

The DIAL technique uses the returns from two different wavelengths that are closely spaced to determine a vertical profile of the number density of a particular molecule. The first wavelength, $\lambda_{\text {on }}$, is the wavelength corresponding to the center of an absorption feature of the molecule of interest while the second wavelength, $\lambda_{\text {off }}$, is the wavelength corresponding to no molecular absorption. From the lidar equation, the DIAL equation is found by considering the return signal from adjacent range bins for both $\lambda_{\text {on }}$ and $\lambda_{\text {off }}$. For $\lambda_{\text {on }}$ and $\lambda_{\text {off }}$ closely spaced so that the atmospheric extinction, backscatter, and receiver efficiency are equal for the two wavelengths, the DIAL equation can be written as

$$
\begin{aligned}
N(r)= & \frac{1}{2\left[\sigma\left(\lambda_{\text {on }}, r\right)\right]-\left[\sigma\left(\lambda_{\text {off }}, r\right)\right] \Delta r} \\
& \times\left\{\ln \left[\frac{P\left(\lambda_{\text {on }}, r\right) P\left(\lambda_{\text {off }}, r+\Delta r\right)}{P\left(\lambda_{\text {on }}, r+\Delta r\right) P\left(\lambda_{\text {off }}, r\right)}\right]\right\} .
\end{aligned}
$$

\section{b. Absorption line selection}

The DIAL instrument described in this paper is focused on measuring water vapor profiles in the lower troposphere using a ground-based instrument. Accurate measurements of water vapor profiles require care in selecting an absorption line that is insensitive to variations in the atmospheric temperature (Machol et al. 2004; Obland 2007; ORNCS; Browell et al. 1991). The evaluation of the temperature sensitivities of water vapor absorption lines in the 820-840-nm spectral region was studied (Obland 2007; ORNCS) using the methods presented by Browell et al. (1991), leading to the selection of a water vapor absorption line for number density profiles.

The temperature dependence of the line strength $S$ associated with an absorption feature is give by (Browell et al. 1991)

$$
\begin{aligned}
S(T)= & S_{0}\left(\frac{T_{0}}{T}\right)^{1.5}\left[\frac{1-\exp \left(-h c \nu_{0} / k T\right)}{1-\exp \left(-h c \nu_{0} / k T_{0}\right)}\right] \\
& \times \exp \left[\frac{h c}{k}\left(\frac{1}{T_{0}}-\frac{1}{T}\right) E^{\prime \prime}\right],
\end{aligned}
$$

where $S_{0}$ is the reference line strength at temperature $T_{0}, T$ is the temperature at which the line strength $S(T)$ is being calculate, $h$ is the Planck constant, $c$ is the speed of light, $k$ is the Boltzmann constant, $\nu_{0}$ is the line center position, and $E^{\prime \prime}$ is the ground state energy of the transition. The absorption cross section $\sigma(T)$ is related to the temperature-dependent line strength through (Browell et al. 1991)

$$
\begin{aligned}
\sigma(T)= & S(T) \frac{\ln (2)}{\pi^{3 / 2}} \frac{\gamma_{L}}{\gamma_{D}} \\
& \times \int_{-\infty}^{\infty} \frac{e^{-t^{2}}}{\left(\frac{\gamma_{L}}{\gamma_{D}}\right)^{2} \ln (2)+\left\{\frac{v-v_{0}}{\gamma_{D}}[\ln (2)]^{1 / 2}-t\right\}^{2}} d t,
\end{aligned}
$$

where $\gamma_{L}=\gamma_{0}\left(P / T_{0}\right)\left(T / T_{0}\right)^{\alpha}$ is the Lorentz line width at temperature $T$ and pressure $P$ associated with pressure broadening, which is dominant in the lower atmosphere below $\sim 2 \mathrm{~km} ; \gamma_{0}$ is the Lorentz line width at temperature $T_{0}$ and pressure $P_{0} ; \alpha$ is the linewidth temperature-dependent parameter; $\gamma_{D}=\left(v_{0 / c}\right)$ $\left([2 k T \ln (2)] / m^{\prime}\right)^{1 / 2}$ is the Doppler line width associated with Doppler broadening, which is important in the upper atmosphere above $\sim 3-4 \mathrm{~km} ; m^{\prime}$ is the mass of the water molecule; and $v$ is the online laser frequency. The absorption cross section in Eq. (5) is calculated based on a Voigt profile, which is a convolution of the Lorentz and Doppler line shapes associated with the water vapor absorption feature. Values of $\gamma_{0}, S_{0}$, and $E^{\prime \prime}$ were 
obtained from the High-Resolution Transmission Molecular Absorption (HITRAN 2000) database (Rothman et al. 2003).

The temperature sensitivity for the number density measurement is calculated using the expression (Browell et al. 1991)

$$
\frac{1}{\sigma} \frac{d \sigma}{d T} \approx \frac{1}{T-T^{\prime}} \frac{\sigma(T)-\sigma\left(T^{\prime}\right)}{\left[\frac{\sigma(T)+\sigma\left(T^{\prime}\right)}{2}\right]},
$$

where $\sigma(T)$ is found using Eqs. (4) and (5). A plot of the number density error as a function of temperature for various ground-state energies is shown in Figs. $1 \mathrm{a}$ and $1 \mathrm{~b}$ for pressures of 1 and $0.25 \mathrm{~atm}$, respectively. The absorption cross section $\sigma(T)$ is temperature independent at the temperature neutral point when $d \sigma / d T=0$.

The three primary criteria for the selection of the water vapor absorption line include the temperature sensitivity, line strength, and absence of nearby absorption features (Machol et al. 2004; Obland 2007; ORNCS; Repasky et al. 2004). The online wavelength chosen for the water vapor DIAL instrument has a wavelength of $828.187 \mathrm{~nm}(828.0069 \mathrm{~nm})$ in a vacuum (air) while the offline wavelength was chosen to be $828.287 \mathrm{~nm}$ $(828.1069 \mathrm{~nm})$ in a vacuum (air). The temperature neutral point for this absorption feature, as seen in Fig. 1, for the ground-state energy of $212.2 \mathrm{~cm}^{-1}$ ranges from $275 \mathrm{~K}$ at $0.25 \mathrm{~atm}$ to $350 \mathrm{~K}$ at $1 \mathrm{~atm}$. At a temperature of $296 \mathrm{~K}$ and a pressure of $1 \mathrm{~atm}$, the line strength associated with the water vapor absorption feature used for the DIAL measurements presented in this paper is $S=1.477 \times$ $10^{-23} \mathrm{~cm}^{-1}\left(\mathrm{~mol} \mathrm{~cm}^{-2}\right)^{-1}$, the full width at half maximum line width is $0.1937 \mathrm{~cm}^{-1}$, and the ground-state energy is $E^{\prime \prime}=212.2 \mathrm{~cm}^{-1}$ (Rothman et al. 2003).

\section{DIAL instrument}

\section{a. DIAL laser transmitter}

A schematic of the DIAL instrument is shown in Fig. 2. A tunable external cavity diode laser (ECDL) is used to injection seed a tapered SOA. The ECDL was constructed in the Littman-Metcalf cavity configuration (Littman and Metcalf 1978; Switzer 2007). A 150-mW diode laser with a center wavelength of $830 \mathrm{~nm}$ (SDL5421 ) is collimated using an aspheric lens with a focal length of $4.5 \mathrm{~mm}$ and a numerical aperture of 0.55 (Thor Laboratories 350230-B). The collimated light is next incident on a 1600 line $\mathrm{mm}^{-1}$ grating $15 \mathrm{~mm}$ wide by 60 $\mathrm{mm}$ long by $10 \mathrm{~mm}$ thick (Spectrogon) at a grazing angle of $3^{\circ}$. The first-order reflection from the diffraction grating is found from $\cos \theta_{\text {out }}=\cos \theta_{\text {in }}-(\lambda / d)$ to be $109^{\circ}$, where $\theta_{\text {in }}\left(\theta_{\text {out }}\right)$ is the angle between the incoming (outgoing) beam and the plane of the diffraction grating, $\lambda$ is the wavelength, and $d$ is the line spacing of the diffraction grating. The first-order reflection is next incident on a roof prism that directs the light back into the diode laser via a second reflection from the diffraction grating. The external cavity has a free spectral range of $3.9 \mathrm{GHz}$ corresponding to a $3.8-\mathrm{cm}$-long external cavity.

The roof prism rotates so that the cavity length changes in concert with the wavelength of light fed back to the diode laser, allowing for mode-hop free tuning. The roof prism can be rotated mechanically by a 3/16100 screw for coarse tuning. Fine rotation of the roof prism is achieved by applying a voltage to a piezoelectric stack (Thor Laboratories AE0505D16) giving modehop free tuning of over $20 \mathrm{GHz}$ at a fixed temperature.

The ECDL is placed on a thermoelectric cooler (TEC) for temperature stabilization. A $10-\mathrm{k} \Omega$ thermistor is used to monitor the temperature of the ECDL, and a commercial temperature controller (ILX LDD3722) is used to stabilize the temperature to within $0.1^{\circ} \mathrm{C}$. A commercial current controller (ILX LDD3722) is used to supply a drive current to the diode laser. The ECDL is operated in a continuous-wave $(\mathrm{cw})$ mode of operation and its performance is summarized as follows. The coarse tuning ranges from 824 to $841 \mathrm{~nm}$ with a modehop free-tuning range of greater than $20 \mathrm{GHz}$ and the full-width half-maximum line width being less than 200 $\mathrm{kHz}$. The maximum output power is $20 \mathrm{~mW}$ with a sidemode suppression of greater than $45 \mathrm{dBm}$. A picture of the ECDL is shown in Fig. 3.

The output of the ECDL is sent through a Faraday isolator to prevent unwanted feedback from affecting its performance. After the Faraday isolator, the light is next incident on a wave plate and polarizing beamsplitter (PBS). The light rejected by the PBS is used to monitor the $\mathrm{cw}$ output of the ECDL, while the light passing through the PBS is used to seed an optical preamplifier. The ratio of the light passing through the PBS to the light rejected by the PBS can be controlled by rotating the wave plate.

The output from the ECDL has the narrow line width and broad tunability needed for the DIAL transmitter but has low cw output power. To increase the optical power while maintaining the spectral properties of the ECDL, the ECDL is used to injection seed an SOA. Since the output of the ECDL was unable to saturate the gain of the SOA, a preamplifier was first used (Sacher Lasertechnik TA830). The output of the preamplifier passed through a Faraday isolator and was then used to injection seed a second SOA (Sacher Lasertechnik TA830). The second SOA produced a cw output power of up to $500 \mathrm{~mW}$. The output of the second SOA was then incident on an acousto-optic modulator (AOM; 

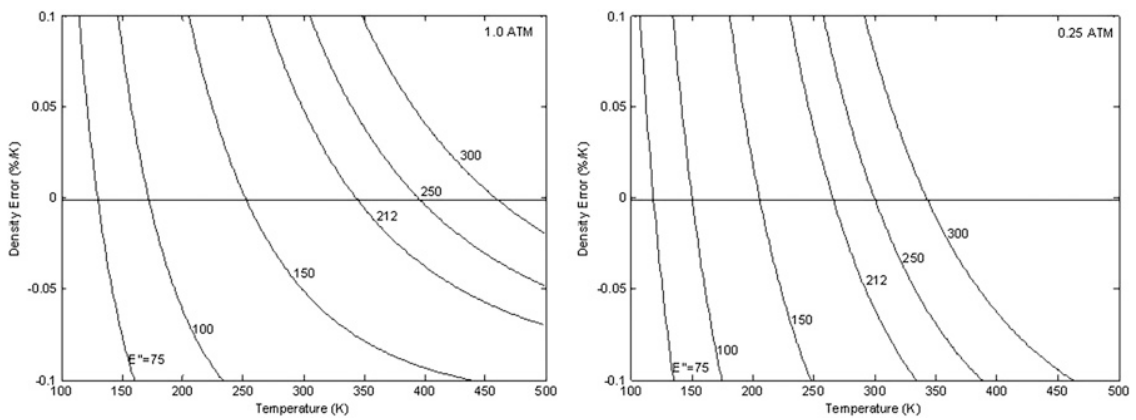

FIG. 1. Temperature sensitivity of water vapor DIAL number density measurement errors at 1.0 - and 0.25 -atm pressure for a range of $E^{\prime \prime}$ values.

Isomet 1205C-2). The first-order diffraction from the AOM was used to create a $1-\mu$ s pulsed output with a $20-\mathrm{kHz}$ pulse repetition frequency for the DIAL instrument, yielding a pulse energy and average power of approximately $0.125 \mu \mathrm{J}$ and $2.5 \mathrm{~mW}$, respectively. The low output pulse energies of the DIAL transmitter are attributed to inefficient coupling into the first-order diffraction out of the AOM. After the AOM, a wedged window was used to direct a small portion of the output beam to a reference energy detector. The minimum divergence of the output of the DIAL transmitter was calculated to be approximately $0.1 \mathrm{mrad}$, where atmospheric turbulence such as scintillation has the potential to increase the divergence drastically.

A plot of the optical power as a function of wavelength for the DIAL laser transmitter is shown in Fig. 4. This plot shows the DIAL laser transmitter can be tuned from 824 to $841 \mathrm{~nm}$, a $17-\mathrm{nm}$ tuning range. The laser transmitter is capable of accessing many water vapor absorption lines; however, care must be taken in choosing an appropriate absorption line to obtain accurate water vapor profiles as discussed in section $2 \mathrm{~b}$ above.

The requirements for DIAL measurements with an error due to individual laser properties of less than 3\% are stated in Bosenberg (1998). These properties arise due to the fact that the absorption cross sections of the water vapor absorption lines are highly dependent on the laser wavelength. If the laser line width, frequency stability, and spectral purity are not well known, errors will arise in the water vapor retrievals owing to uncertainties in the actual cross section of the water vapor associated with transmitted laser light. The requirements for the laser transmitter for an error of less than $3 \%$ from individual laser properties include a line width of less than $298 \mathrm{MHz}$, a spectral purity of greater than 0.995 , and a frequency stability of better than \pm 160 $\mathrm{MHz}$ (Bosenberg 1998). The measured laser transmitter properties include a line width of less than 0.300 $\mathrm{MHz}$, a spectral purity of 0.995 , and a frequency sta- bility of $\pm 88 \mathrm{MHz}$ (Obland 2007; ORNCS; Nehrir et al. 2008; Obland et al. 2007). Thus, the laser transmitter meets the requirements needed for accurate water vapor retrievals.

\section{b. DIAL receiver}

A schematic of the DIAL receiver is shown in Fig. 5. Light scattered by the atmosphere is collected using a commercial f/10 Schmidt-Cassegrain telescope (Celestron CGE1100) with a 28 -cm-diameter primary mirror, which gives a nominal effective focal length of $2.8 \mathrm{~m}$ for the telescope. Light collected by the telescope passes through a focus and is then incident on a collimating lens with a focal length of $10 \mathrm{~cm}$. The collimated beam diameter is approximately $1.5 \mathrm{~cm}$. The collimated light passes through a narrowband filter with a $65 \%$ transmission at a center wavelength of $828.06 \mathrm{~nm}$ and a fullwidth at half-maximum line width of $0.2 \mathrm{~nm}$. After the narrowband filter, the light is focused using a focusing lens with a focal length of $2.5 \mathrm{~cm}$. The focused light is

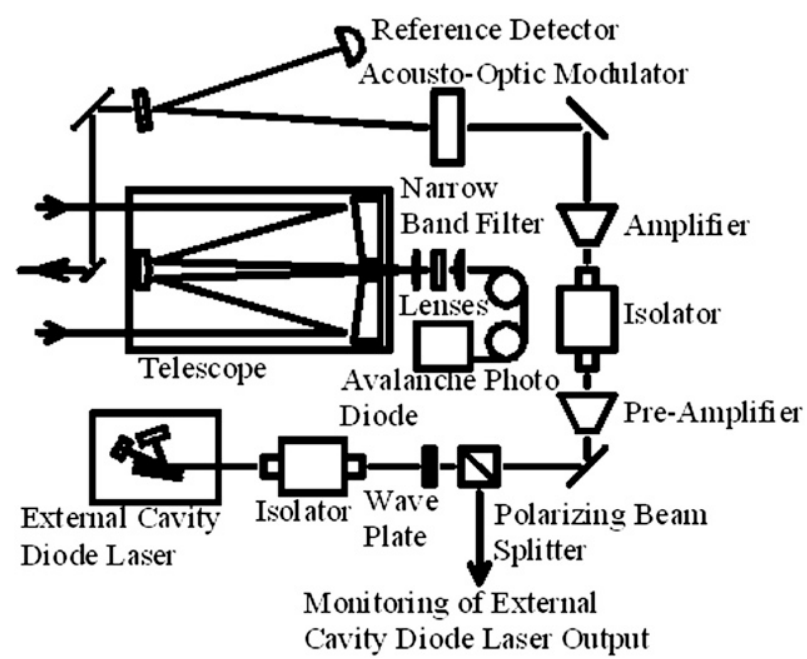

FIG. 2. The DIAL instrument used for studying water vapor number densities in the lower troposphere. 


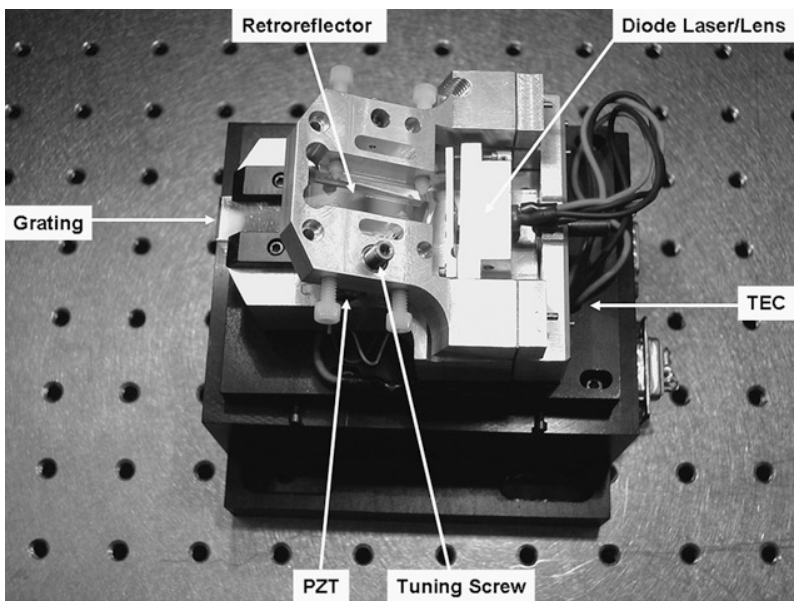

FIG. 3. The ECDL built in the Littman-Metcalf external-cavity configuration used to injection seed cascaded flared amplifiers used for the DIAL transmitter. The major components of the ECDL are identified along with the pivot point about which the retroreflecting roof prism rotates: thermoelectric cooler (TEC) and piezoelectric transducer (PZT).

launched into a multimode fiber with a core diameter of $105 \mu \mathrm{m}$ and a numerical aperture of NA $=0.22$ that acts as the system field stop and yields a full-angle field of view of $37.5 \mu \mathrm{rad}$. The multimode optical fiber is the smallest aperture in the DIAL receiver, therefore alleviating the need for an iris as the field stop at the focal plane of the telescope, which has a minimum closing aperture of $1 \mathrm{~mm}$, yielding a relatively large full-angle field of view of $357 \mu \mathrm{rad}$. A fiber-coupled photoncounting avalanche photodiode (Perkin-Elmer SPCMAQR-13-FC) module is used to detect the collected optical signal, yielding a far-field full-angle field of view of $150 \mu \mathrm{rad}$. This field of view is 4 times greater than the field of view imposed by the field stop due to the magnification of the image of the field of view at the multimode fiber as a result of the collimating and focusing lenses used in the receiver. The receiver optics were chosen so that the $f$ numbers of the telescope and collimating lens are matched. The collimated light is needed because the transmission of the narrowband optical filter is strongly dependent on the angle of incidence of the light on this filter. The focusing lens was chosen so that the $f$ number associated with this lens matched the $f$ number associated with the multimode optical fiber used to deliver the received light to the APD.

\section{c. Data collection}

Automated control and data acquisition for the DIAL instrument is achieved using the Labview programming environment on a portable laptop computer. The data acquisition software works in the follow manner. The laser transmitter is first tuned onto a user-defined wave-

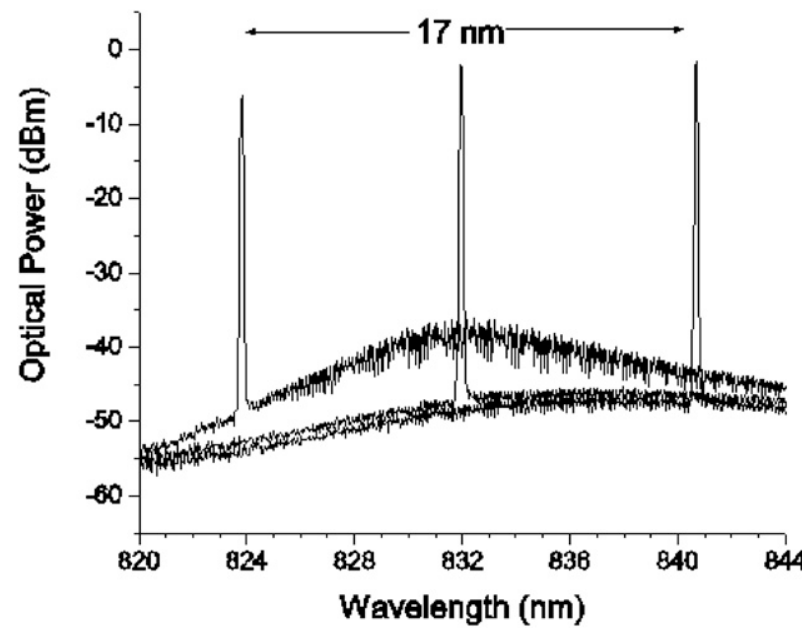

FIG. 4. Output power as a function of wavelength for the injection-seeded tapered amplifier. Good side mode suppression ratio and high optical power are maintained through the amplifier while still maintaining the spectral properties of the seed laser.

length that corresponds to the peak absorption of a water vapor line at $828.0069 \mathrm{~nm}(828.187 \mathrm{~nm})$ in air (in a vacuum). The wavelength of the laser transmitter is monitored using the light rejected from the first PBS shown in Fig. 2 using a Burleigh wavemeter (WA-1500), which has a frequency resolution of $\pm 88 \mathrm{MHz}$. The wavelength is polled every $2 \mathrm{~s}$ over a general-purpose interface bus (GPIB) and an appropriate voltage correction is made to the ECDL's piezoelectric transducer using a programmable voltage source until the laser

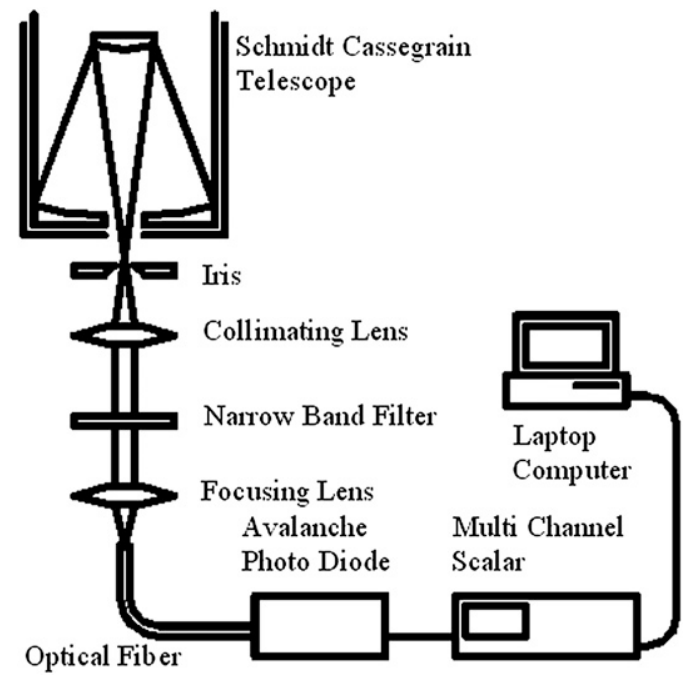

FIG. 5. The DIAL receiver. The $f$ number of the telescope and the $f$ number of the fiber coupling lens are matched to that of the collimating lens and the multimode fiber, respectively, to yield a far-field full field of view of $150 \mu \mathrm{rad}$. 
transmitter achieves a steady-state wavelength that is within $\pm 88 \mathrm{MHz}$ of the selected wavelength. Next, an arbitrary waveform generator (AWG) is used to begin data collection from the APD and the multichannel scalar card (MCS) for $5 \mu$ s with the output from the laser transmitter turned off to obtain background measurements used for background subtraction during postmeasurement data processing. After the 5- $\mu$ s delay used for background measurements, the AWG triggers the AOM, creating a $1-\mu$ s pulse corresponding to a $300-\mathrm{m}$ range bin at a $20-\mathrm{kHz}$ pulse repetition rate, resulting in a $2 \%$ duty cycle for the first-order diffracted beam from the AOM. During the $6-\mu$ s delay between the initial trigger and the end of the laser pulse, the MSC samples the APD data across 120 bins, each of width 50 ns. The subsequent return signal resulting from the laser interaction with the atmosphere accounts for the remaining 380 bins sampled by the MSC. These 380 bins correspond to $19 \mu$ s of return data. This process is repeated 25 $\mu$ s later and averaged 20000 times over $1 \mathrm{~s}$, yielding 400 range-resolved range bins of $7.5 \mathrm{~m}$ each, corresponding to an altitude of $3.0 \mathrm{~km}$. The data collection process is repeated for the online wavelength of $828.0069 \mathrm{~nm}$ (828.187) in air (in a vacuum) for a user-defined period of time, normally $60 \mathrm{~s}$. The software then tunes the laser to the offline wavelength of $828.1069 \mathrm{~nm}$ (828.287) in air (in a vacuum) and data are collected for a user-defined time, normally $60 \mathrm{~s}$. This entire process is repeated for approximately $1 \mathrm{~h}$ so that sufficient returns are obtained to begin calculating the range-resolved water vapor number density profile using the DIAL equation.

Once the data are obtained, MATLAB programs are used to analyze the raw returns. First, spectral filtering is achieved by analyzing the polled wavelength from the wavemeter so that only the data that meet the \pm 160 $\mathrm{MHz}$ of the selected wavelength requirements are considered for the water vapor profile measurement. Once the desired DIAL raw returns are obtained, the data are spatially averaged into sets of 20 bins such that two data points are obtained for each laser pulse according to the highest resolution defined by the transmitter pulse width of $300 \mathrm{~m}$ for the $1-\mu \mathrm{s}$ pulse. Next, the raw returns are normalized by the reference power measurements and averaged on a second-tosecond basis to account for the shot-to-shot laser intensity fluctuations. Finally, the spatially averaged background measurements obtained from the first $5 \mu$ s of data collected before each laser pulse are used to subtract background light counts from each range bin, yielding a plot of the range-resolved online and offline return signals. A plot of the background-subtracted counts as a function of range from nighttime data taken on 9 March 2008 is shown in Fig. 6. The open and closed circles represent the online and offline counts measured by the DIAL instrument, respectively.

The processed atmospheric returns shown in Fig. 6 are used with collocated radiosonde-derived temperature and pressure measurements to calculate water vapor number density profiles using the DIAL equation presented in Eq. (3). The only unknowns in the DIAL equation needed to calculate the water vapor number densities are the online and offline absorption cross sections $\sigma$ at a given range $r$. The temperature profile obtained from the radiosonde measurements are then used in Eqs. (4) and (5) along with the ground-state energy transition $E^{\prime \prime}$ for the water vapor absorption obtained from the HITRAN database (Rothman et al. 2003) to calculate the water vapor line absorption cross section as a function of range. Using the range-dependent online and offline cross sections together with the data shown in Fig. 6, Eq. (3) can be used to calculate the water vapor number density as a function of range as shown in Fig. 7. The water vapor number densities measured using the DIAL instrument show good agreement with the collocated radiosonde measurements above approximately $700 \mathrm{~m}$. Below $700 \mathrm{~m}$, three dominant effects limit the accuracy of the DIAL measurement. First, because the laser beam and telescope field of view are coaxial in the DIAL instrument, the central obscuration of the telescope shields the telescope from the large near-field returns. Second, due to the large scattering angles of the near-field returns subtended upon the receiver's collimation optics, sufficient coupling is not achieved into the optical fiber. Finally, because the two previous effects are equal for both the online and offline wavelengths and cancel out in the DIAL equation, the deviations of the measured results using the DIAL instrument and the radiosonde below $700 \mathrm{~m}$ are best described by an angle-dependent wavelength detuning of the narrowband filter that is discussed in section 4 .

\section{DIAL correction factor}

The center wavelength $\lambda$ for a narrowband filter shifts to shorter wavelengths as the angle of incidence $\theta$ of the incoming light increases. The center wavelength of the narrowband filter $\lambda^{\prime}$ can be written as (Hansch 1972)

$$
\lambda^{\prime}=\lambda_{0} \cos \left(\frac{\theta}{n}\right)
$$

where $\lambda_{0}$ is the center wavelength of the filter at $\theta=0$ and $n$ is the index of refraction of the narrowband filter. The maximum angle incident on the filter can be calculated by considering the geometry of the receiver as shown schematically in Fig. 8. While the telescope has a 


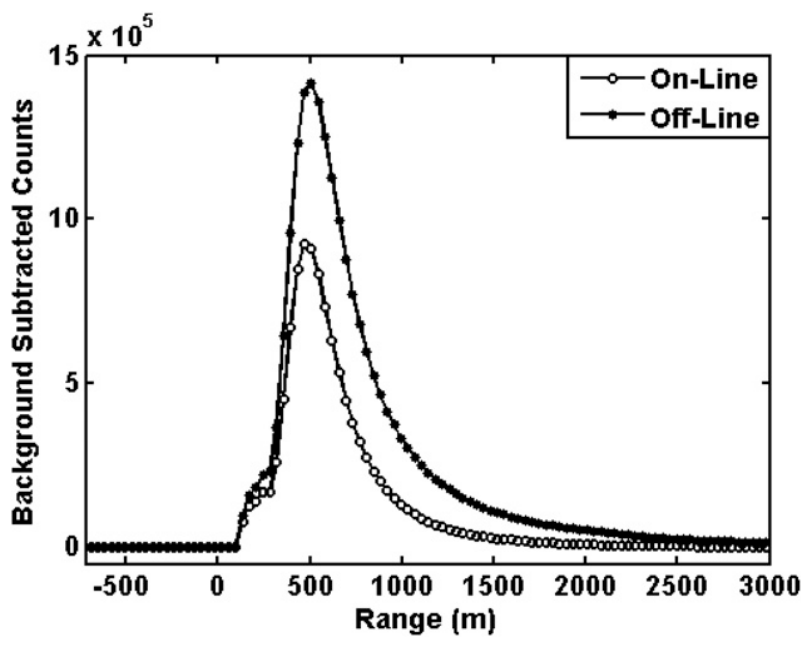

FIG. 6. The background subtracted atmospheric returns as a function of range. Sufficient overlap between the DIAL transmitter and receiver yields measurable atmospheric returns up to $2.5 \mathrm{~km}$. The attenuated returns at the online wavelength are attributed to water vapor absorption through the lower troposphere. These measurements were taken starting at 2109 LT 9 Mar 2008 and calculated using $150-\mathrm{m}$ vertical range bins, which were averaged over $67 \mathrm{~min}$.

nominal focal length of $2.8 \mathrm{~m}$, our measurements indicate the effective focal length is $f_{t}=2.807 \mathrm{~m}$. Using the thin lens equation, a scattering center at a distance $r$ will produce an image formed by the telescope lens at a distance $d_{1}$ from the effective lens:

$$
d_{1}=\frac{r f_{t}}{r-f_{t}} .
$$

Using this image as the object for the second collimating lens with a focal length $f_{c}$, the object distance from the collimating lens is found as

$$
d_{2}=f_{t}+f_{c}+\delta-d_{1},
$$

where the effective lens of the telescope and the collimating lens are separated by $f_{t}+f_{c}+\delta$. For the DIAL instrument described in this paper, $\delta \sim 2 \mathrm{~cm}$. With $\delta=2$ $\mathrm{cm}$, light from a point object at $r=300 \mathrm{~m}$ will pass through the filter with $\theta=0$. Objects closer or farther than $r=300 \mathrm{~m}$ will pass through the filter with $\theta \neq 0$ and will experience a change in transmission due to a change in the angle. The image at $d_{2}$ will create an image associated with the collimating lens at

$$
d_{3}=\frac{d_{2} f_{c}}{d_{2}-f_{c}} .
$$

Finally, using the small angle approximation, the maximum angle of incidence for a ray incident on the narrowband filter $\theta_{\max }$ is

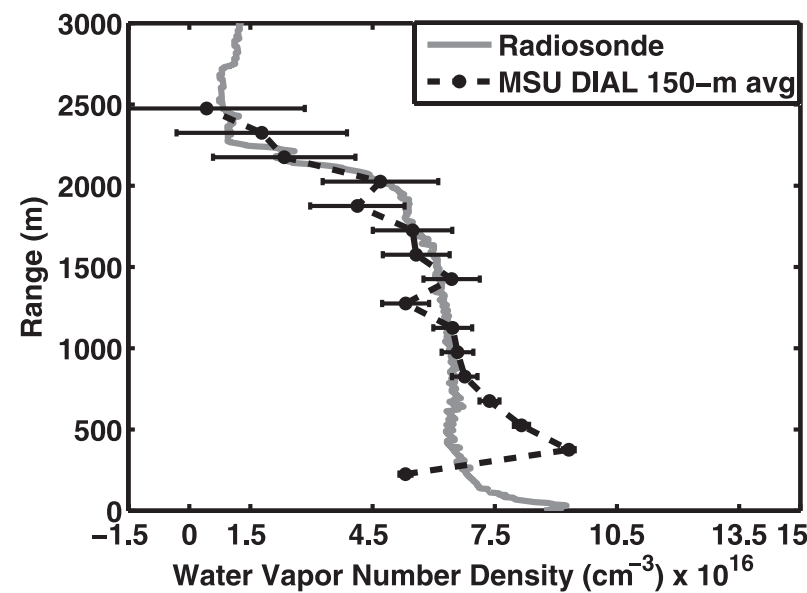

FIG. 7. The vertical water vapor number density profile recorded starting at 2109 LT 9 Mar 2008 using the atmospheric returns obtained from Fig. 6.

$$
\theta_{\max }=\frac{R}{d_{3}}
$$

where $R$ is the radius of the nominally collimated beam seen after the collimating lens. A value of $R=0.75 \mathrm{~cm}$ was measured for the DIAL instrument described in section 3 .

The narrowband filter has a center wavelength of $828.01 \pm 0.05 \mathrm{~nm}$ and a half-width at half-maximum (HWHM) value of $\Delta \lambda=0.125 \pm 0.025 \mathrm{~nm}$. The transmission as a function of wavelength, $T(\lambda)$, has a Lorentzian profile that can be written as

$$
T(\lambda)=T_{0} \frac{\Delta \lambda^{2}}{\Delta \lambda^{2}+\left(\lambda-\lambda^{\prime}\right)^{2}},
$$

where $T_{0}=0.65$ is the maximum transmission of the narrowband filter. A plot of the narrowband optical filter transmission as a function of wavelength is shown in Fig. 9. The online $\lambda_{\text {on }}$ and offline $\lambda_{\text {off }}$ wavelengths for the water vapor DIAL are also plotted in Fig. 9. Taking into

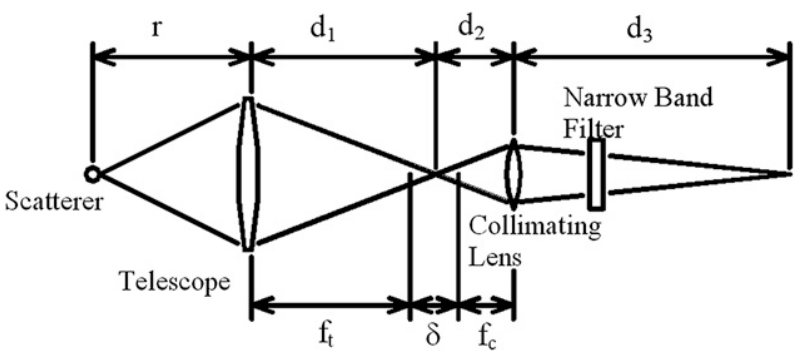

FIG. 8. Geometrical schematic of the DIAL receiver. Misplacement of the collimation optic behind the focal plane of the telescope, denoted as $\delta$, results in wavelength detuning of the narrowband filter and results in false DIAL measurements. 


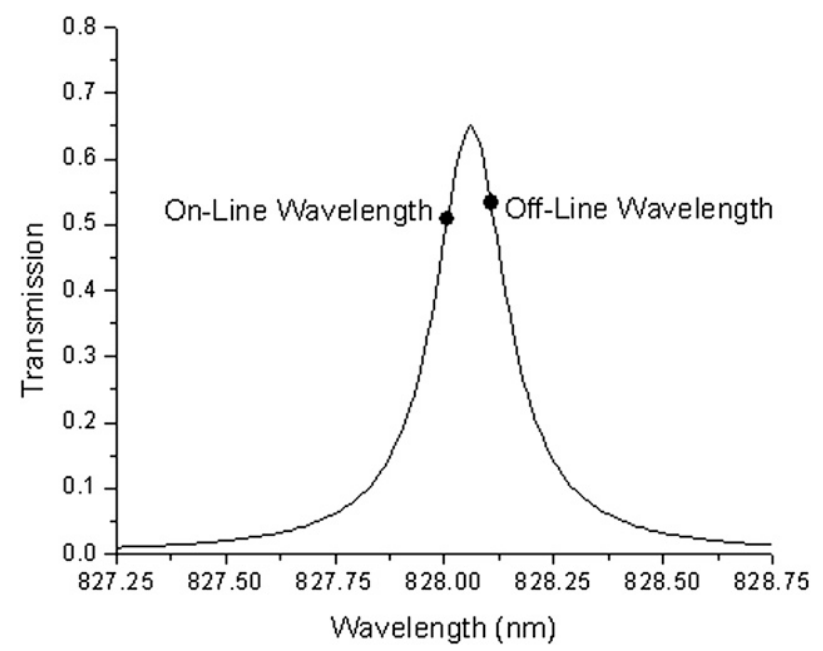

FIG. 9. The narrowband optical filter transmission as a function of wavelength. The online and offline wavelengths in air for the water vapor DIAL instrument are also indicated.

account the angle tuning of the filter as a function of $\theta$, Eq. (7) can be substituted into Eq. (12) using a small angle approximation to yield the following filter transmission as a function of wavelength and angle of incidence:

$$
T(\lambda, \theta)=T_{0} \frac{\Delta \lambda^{2}}{\Delta \lambda^{2}+\left(\lambda-\lambda_{0}-\frac{\lambda_{0} \theta^{2}}{2 n^{2}}\right)^{2}} .
$$

The effective transmission $T_{\text {eff }}$ can then be found from

$$
T_{e f f}(\lambda)=\frac{1}{\theta_{\max }} \int_{0}^{\theta_{\max }} T(\lambda, \theta) d \theta .
$$

The effective transmission found in Eq. (14) is a function of range $r$ since the maximum angle of incidence $\theta_{\max }$ is a function of range $r$.

The effective transmission as a function of range for the water vapor DIAL instrument described in section 3 can be found using Eqs. (7)-(14). For these calculations, $\lambda_{0}=828.06 \mathrm{~nm}, \Delta \lambda=0.1 \mathrm{~nm}, f_{t}=2.807 \mathrm{~m}, f_{c}=10 \mathrm{~cm}$, $\delta=2.0 \mathrm{~cm}$, and $n=1.4$. A plot of the maximum angle and the effective transmission for the online and offline wavelengths is shown in Fig. 10. Using these results, the DIAL equation can be written as

$$
N(r)=\frac{1}{2\left[\sigma\left(\lambda_{\text {on }}, r\right)\right]-\left[\sigma\left(\lambda_{\text {off }}, r\right)\right] \Delta r}\left\{\ln \left[\frac{P\left(\lambda_{\text {on }}, r\right) P\left(\lambda_{\text {off }}, r+\Delta r\right)}{P\left(\lambda_{\text {on }}, r+\Delta r\right) P\left(\lambda_{\text {off }}, r\right)}\right]-\ln \left[\frac{T_{F}\left(\lambda_{\text {on }}, r\right) T_{F}\left(\lambda_{\text {off }}, r+\Delta r\right)}{T_{F}\left(\lambda_{\text {on }}, r+\Delta r\right) T_{F}\left(\lambda_{\text {off }}, r\right)}\right]\right\}
$$

where the correction factor $T_{\text {corr }}(r)$ is defined as

$$
T_{\text {corr }}(r)=-\ln \left[\frac{T_{F}\left(\lambda_{\text {on }}, r\right) T_{F}\left(\lambda_{\text {off }}, r+\Delta r\right)}{T_{F}\left(\lambda_{\text {on }}, r+\Delta r\right) T_{F}\left(\lambda_{\text {off }}, r\right)}\right]
$$

and is plotted as a function of range in Fig. 11. Note that for $r=300 \mathrm{~m}$ in Fig. 11, the correction factor is approximately zero; hence, a zero incidence angle at the narrowband filter at this altitude can be seen in Fig. 10 as expected from the lens separation mentioned earlier. The correction factor affects the DIAL equation mostly for lower elevations where the light collected by the DIAL receiver experiences a larger maximum angle of incidence at the narrowband filter that affects the overall filter transmission.

A plot of the water vapor profile using the DIAL equation presented in Eq. (15) with the correction factor $T_{\text {corr }}$ calculated above is shown in Fig. 12. The solid line represents the water vapor profile measured using a collocated radiosonde while the circles connected by the dotted line represent the measurements made using the DIAL instrument. For these data, the radiosonde temperature and pressure profiles were used to calculate the absorption cross section of the water vapor. Good agree- ment between the expected and measured profiles is seen between 225 and $2500 \mathrm{~m}$.

\section{Discussion}

The goal of the water vapor DIAL instrument is to autonomously collect nighttime and daytime water vapor profiles using a ground-based instrument. In the absence of radiosonde measurements, ideal temperature and pressure profiles in the lower troposphere can be estimated using the surface temperature $T_{s}$ and surface pressure $P_{s}$ as demonstrated previously by Machol et al. (2004). Using these modeled temperature and pressure profiles, a water vapor profile was measured where the results showed good agreement to within $2 \%$ of a collocated radiosonde measurement. Other methods of acquiring temperature and pressure profiles in order to calculate the range-dependent absorption cross section needed for calculating water vapor profiles are also being investigated. An attractive candidate for obtaining these measurements uses grid assimilation radiosonde data from local or national weather service sites.

A plot of the water vapor number density as a function of altitude for a second night is shown in Fig. 13. This 


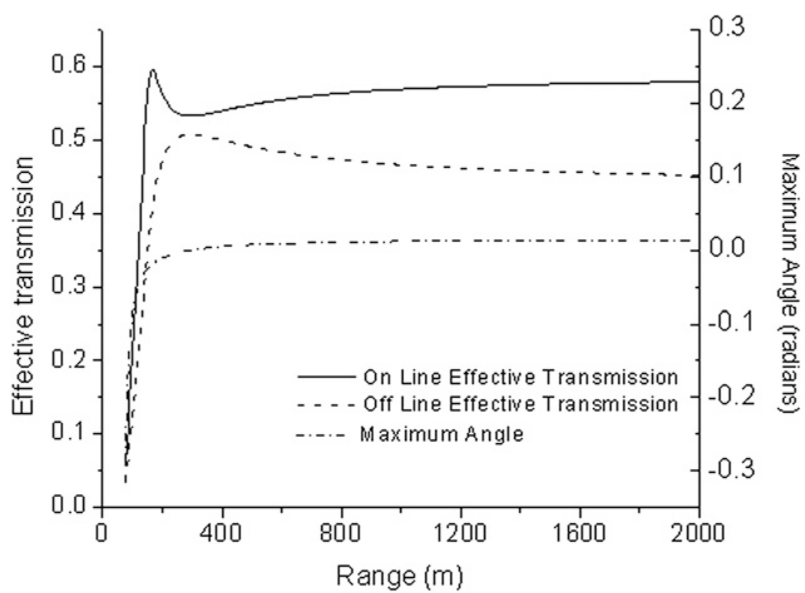

FIG. 10. The maximum incident angle projected onto the narrowband filter along with the effective transmission for the online and offline wavelengths as a function of range.

number density profile was calculated using the DIAL equation with the same correction factor calculated above. The circles connected by the dotted line represent the measurements made using the DIAL instrument while the solid line represents measurements made using a collocated radiosonde. Good agreement between the measured water vapor profile using the DIAL instrument and measurements using the radiosonde are demonstrated over the altitude range of 225-2500 m.

The current DIAL instrument uses a lower pulse energy of $0.125 \mu \mathrm{J}$ with a high pulse repetition frequency of $20 \mathrm{kHz}$ to obtain enough return counts to measure water vapor profiles in the lower troposphere. One important question to be addressed for this instrument is how long of an averaging time is needed to

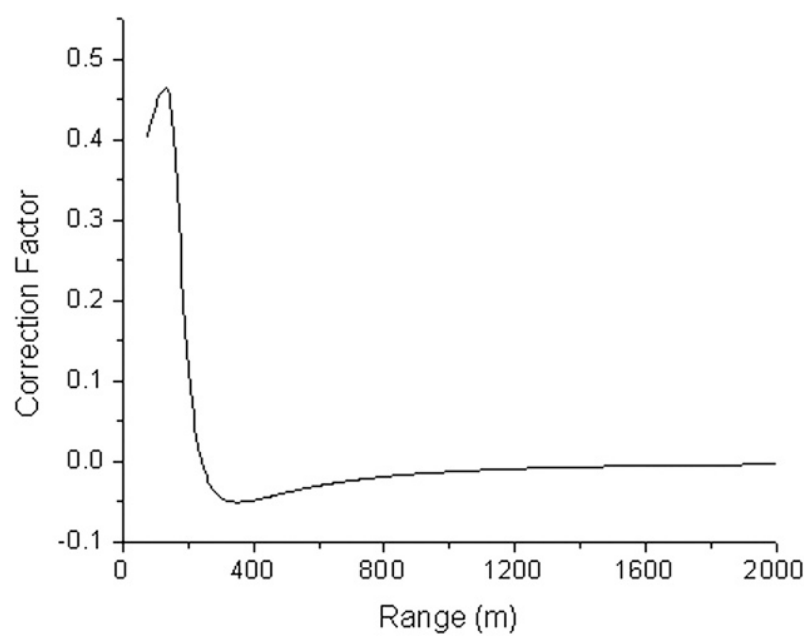

FIG. 11. The correction factor $T_{\text {corr }}(r)$ as a function of range derived using Eqs. (7)-(14).

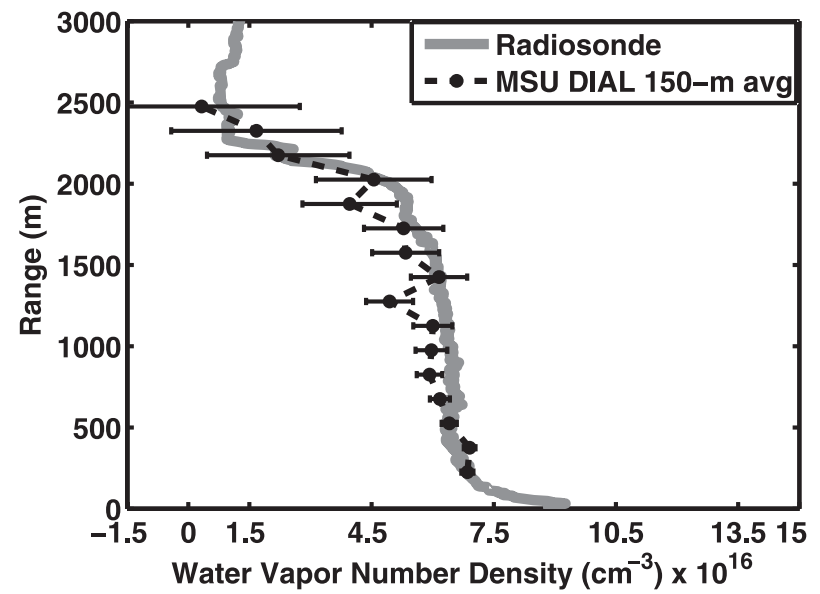

FIG. 12. The water vapor number density profile using the DIAL equation presented in Eq. (15) with the correction factor $T_{\text {corr }}$ included to correct for wavelength detuning in the narrowband filter as a function of altitude. The temperature and pressure profiles measured using the radiosonde were used to calculate the absorption cross section of the water vapor as a function of range. This profile was recorded starting at 2109 LT 9 Mar 2008 using the atmospheric returns obtained from Fig. 6.

obtain a sufficiently accurate nighttime water vapor profile. A plot of the water vapor profile as a function of altitude is shown in Fig. 14 for averaging times ranging from $2 \mathrm{~min}$ ( $1 \mathrm{~min}$ online, $1 \mathrm{~min}$ offline) to $78 \mathrm{~min}$ (33 min online, 45 min offline). The solid circles with the error bars represent the measurements made using the DIAL instrument, while the solid line represents the water

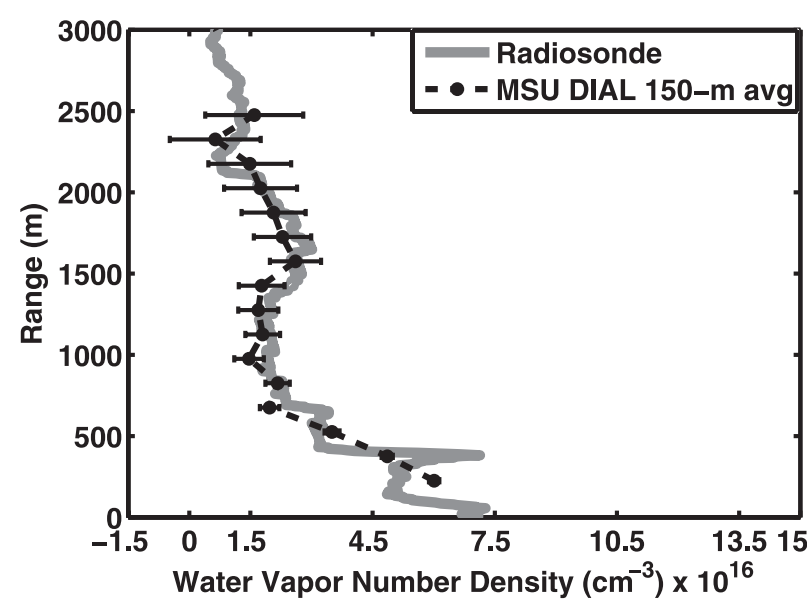

FIG. 13. The water vapor number density as a function of altitude for a second night of data using the same correction factor used in Fig. 12. Surface temperature and surface pressure measurements were used to model and calculate the temperature and pressure profiles used to then calculate the range-dependent absorption cross section for the water vapor. These measurements were taken starting at 2100 LT 19 Feb 2008 and calculated using 150-m vertical range bins, which were averaged over $78 \mathrm{~min}$. 

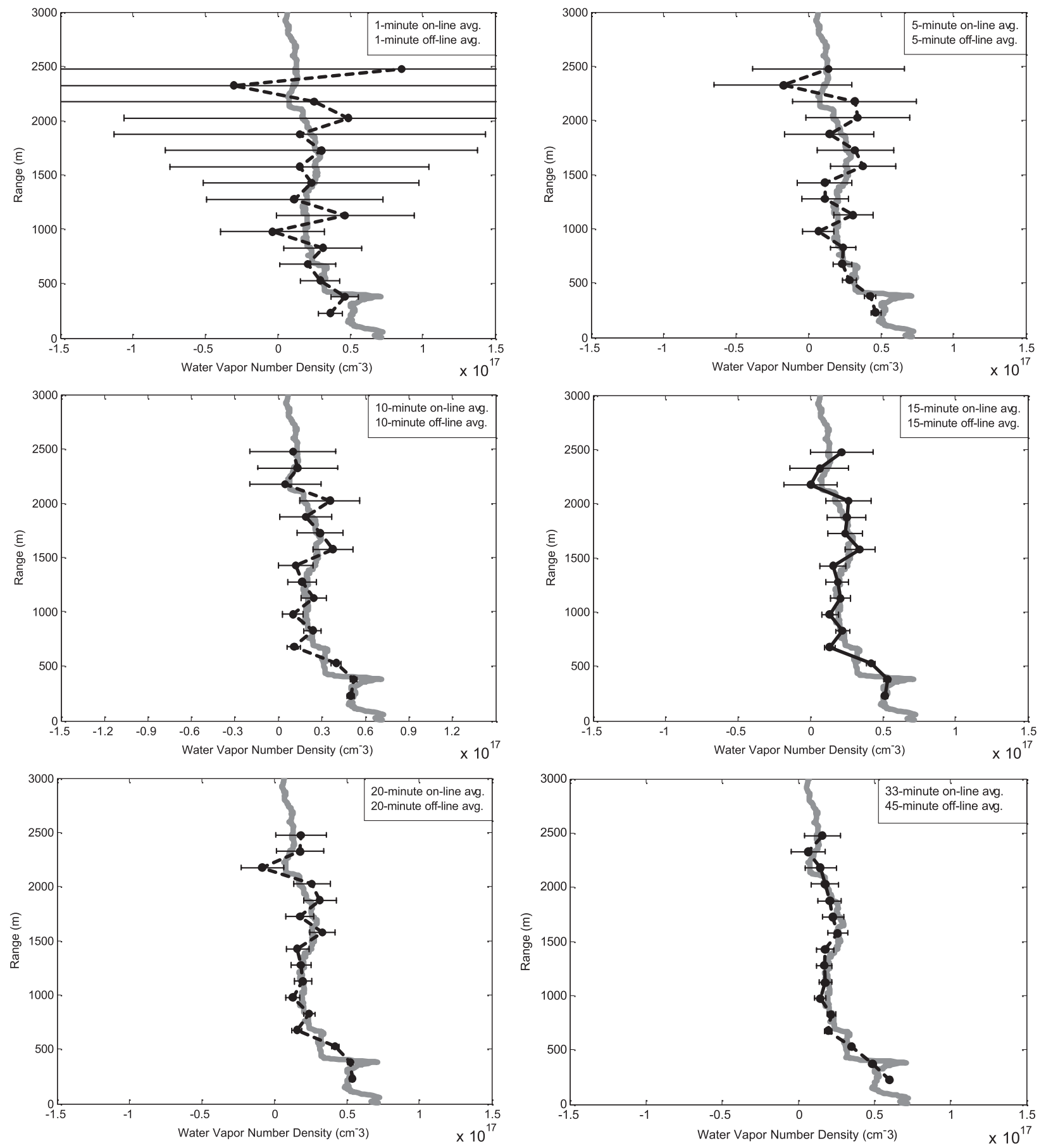

FIG. 14. The water vapor number density profile as a function of altitude for averaging times ranging from $2 \mathrm{~min}$ ( $1 \mathrm{~min}$ online, $1 \mathrm{~min}$ offline) to $78 \mathrm{~min}$ (33 min online, $45 \mathrm{~min}$ offline). These measurements were taken starting at $2100 \mathrm{LT} 19$ Feb 2008 and calculated using 150 -m vertical range bins.

vapor profile measured using the collocated radiosonde. Figure 14 indicates that a reasonable water vapor profile to within a $10 \%$ error relative to the in situ measurement can be obtained with about a 20-min averaging time (10 min online, $10 \mathrm{~min}$ offline). Although the nighttime signal-to-noise ratio (SNR) is directly proportional to the integration period, where larger temporal averaging yields a higher SNR, the same principle does not apply to daytime measurements. Despite having a sufficiently narrow far-field full field of view and 
optical pass band in the DIAL receiver, scattering from the low peak pulse energies are not discernable from the background solar radiation, limiting the DIAL instrument strictly to nighttime operation.

Because the DIAL transmitter exhibits such low pulse energies, longer integration periods are required to retrieve adequate returns to calculate water vapor profiles. Work is currently under way at Montana State University to build a second-generation water vapor DIAL instrument based on custom-built widely tunable ECDLs and SOAs to increase the output pulse energy of the instrument by a factor of 10-20, yielding maximum output pulse energies of $2 \mu \mathrm{J}$ with a pulse repetition frequency of $20 \mathrm{kHz}$, corresponding to a maximum resolvable range of $7.5 \mathrm{~km}$ for the DIAL measurement. This increase in peak pulse energy will allow for daytime measurements as well as shorter integration periods that will provide range-resolved water vapor flux data extending above the boundary layer at time periods approaching those of the lifetimes of atmospheric cycles in the lower troposphere, on the order of 1-2 min.

Improvements over the first-generation DIAL instrument include pulsed operation by directly current pulsing custom-built SOAs, alleviating the need for an AOM, as well as the use of compact inline free-space fiber couplers for signal detection, both of which significantly decrease the overall instrument size on the $2 \mathrm{ft} \times$ $4 \mathrm{ft} \times 2 \mathrm{ft}$ breadboard housing. An alternative to the SOA approach for amplifying the ECDL output is also under investigation. The second approach will utilize an injection-seeded diode-laser-pumped solid-state laser based on a Cr:LiSAF gain medium, allowing for a compact higher-power DIAL transmitter. The lightweight and compact footprint of the DIAL instrument as well as the high wall-plug efficiency of the improved performance of the various candidates for the DIAL transmitter all have the potential to lead to the next generation of widely tunable DIAL instruments that in the future may be acceptable candidates for use in multipoint water vapor lidar networks or satellite arrays used to study cloud formation via water vapor and aerosol interactions.

\section{Conclusions}

A diode-laser-based DIAL instrument for measuring water vapor profiles in the lower troposphere has been built, and tested, and has measured water vapor profiles across several nights that compare favorably with collocated radiosonde measurements. The laser transmitter is based on a widely tunable ECDL capable of accessing any water vapor absorption line in the 824841-nm spectral region, an important capability that has been called for in other studies. Despite the low power limitations of the instrument, water vapor profiles with relatively low error values have been measured using the DIAL instrument and have compared favorably with data gathered from a collocated radiosonde, indicating that accurate water vapor profiles can be measured with an averaging time of approximately $20 \mathrm{~min}$.

Accurate measurements of water vapor profiles, particularly at lower altitudes, require care in using the DIAL equation. In particular, a correction factor has been introduced into the DIAL equation to take into account the effects of the angle of incidence of the collected light on the narrowband filter used in the DIAL receiver. The correction factor plays an important role in light scattered from lower altitudes because this scattered light will have a larger maximum angle of incidence, allowing water vapor profiles to be extended down to approximately $250 \mathrm{~m}$ above the instrument.

Acknowledgments. This work was supported by NASA Grant NNX06AD11G. Partial support was also provided by the NASA Graduate Student Researchers Program (GSRP).

\section{REFERENCES}

Behrendt, A., and Coauthors, 2007: Intercomparison of water vapor data measured with lidar during IHOP_2002. Part I: Airborne to ground-based lidar systems and comparisons with chilled-mirror hygrometer radiosondes. J. Atmos. Oceanic Technol., 24, 3-21.

Bosenberg, J., 1998: Ground based-differential absorption lidar for water vapor and temperature profiling: Methodology. Appl. Opt., 37, 3845-3860.

Browell, E. V., S. Ismail, and B. E. Grossman, 1991: Temperature sensitivity of differential absorption lidar measurements of water vapor in the $720 \mathrm{~nm}$ region. Appl. Opt., 30, 1517-1524.

$\ldots, \ldots$, and W. B. Grant, 1998: Differential absorption lidar (DIAL) measurements from air and space. Appl. Phys., 67B, 399-410.

Dabberdt, W. F., and T. W. Schlatter, 1996: Research opportunities from emerging atmospheric observing and modeling capabilities. Bull. Amer. Meteor. Soc., 77, 305-323.

Divakarla, M. G., C. D. Barnet, M. D. Goldberg, L. M. McMillin, E. Maddy, W. Wolf, L. Zhou, and X. Liu, 2006: Validation of atmospheric infrared sounder temperature and water vapor retrievals with matched radiosonde measurements and forecasts. J. Geophys. Res., 111, D09S15, doi:10.1029/ $2005 J D 006116$.

Ehret, G., C. Kiemle, W. Renger, and G. Simmet, 1993: Airborne remote sensing of tropospheric water vapor with a near infrared differential absorption lidar system. Appl. Opt., 32, 4534-4551.

Feltz, W. F., W. L. Smith, H. B. Howell, R. O. Knuteson, H. Woolf, and H. E. Revercomb, 2003: Near-continuous profiling of temperature, moisture, and atmospheric stability using the Atmospheric Emitted Radiance Interferometer. J. Appl. Meteor., 42, 584-597. 
Goldsmith, J. E. M., F. H. Blair, S. E. Bisson, and D. D. Turner, 1998: Turn key Raman lidar for profiling atmospheric water vapor, clouds, and aerosols. Appl. Opt., 37, 4979-4990.

Hansch, T. W., 1972: Repetitively pulsed tunable dye laser for high resolution spectroscopy. Appl. Opt., 11, 895-898.

Ismail, S., E. V. Browell, R. A. Ferrare, S. A. Kooi, M. B. Clayton, V. G. Brackett, and P. B. Russell, 2000: LASE measurements of aerosol and water vapor profiles during TARFOX. J. Geophys. Res., 105, 9903-9916.

Kovalev, V. A., and W. E. Eichinger, 2004: Elastic Lidar: Theory, Practice, and Analysis Methods. John Wiley and Sons, 615 pp.

Littman, M. G., and H. J. Metcalf, 1978: Spectrally narrow pulsed dye laser without beam expander. Appl. Opt., 17, 2224-2227.

Machol, J. L., and Coauthors, 2004: Preliminary measurements with an automated compact differential absorption lidar for the profiling of water vapor. Appl. Opt., 43, 3110-3121.

Nehrir, A. R., K. S. Repasky, M. D. Obland, Y. Xiong, J. L. Carlsten, and J. A. Shaw, 2008: Water vapor profiling using a compact widely tunable diode laser differential absorption lidar (DIAL). Proc. Int. Laser Radar Conf., Boulder, CO, International Coordination Group on Laser Atmospheric Studies, 1060-1063.

Obland, M. D., 2007: Water vapor profiling using a widely tunable amplified diode laser differential absorption lidar (DIAL) Ph.D. dissertation, Montana State University, 189 pp.

— L. S. Meng, K. S. Repasky, J. A. Shaw, and J. L. Carlsten, 2005: Progress toward a water-vapor differential absorption lidar (DIAL) using a widely tunable amplified diode laser source. Proc. SPIE, 5887, 205-215.

, K. S. Repasky, J. A. Shaw, and J. L. Carlsten, 2006: Preliminary testing of a water-vapor differential absorption lidar (DIAL) using a widely tunable amplified diode laser source. Proc. Int. Geoscience and Remote Sensing Symp., Denver, CO, IEEE, 3949-3952.

_ A. R. Nehrir, K. S. Repasky, J. L. Carlsten, and J. A. Shaw, 2007: Application of extended tuning range for external cavity diode lasers to water vapor differential absorption measurements. Opt. Eng., 46, 084301, doi:10.1117/1.2771654.

Rall, J. A. R., J. B. Abshire, D. Reusser, and M. Humphrey, 1996: Measurements of atmospheric water vapor using a compact AlGaAs laser based DIAL instrument. Conf. on Lasers and Electro-Optics, Anaheim, CA, Optical Society of America, CWD5.
Reagan, J. A., T. W. Cooley, and J. A. Shaw, 1993: Prospects for an economical, eye safe water vapor lidar. Proc. Int. Geoscience and Remote Sensing Symp., Tokyo, Japan, IEEE, 872-874.

—, H. Liu, and J. F. McCalmont, 1996: Laser diode based new generation lidars. Proc. Int. Geoscience and Remote Sensing Symp., Lincoln, NE, IEEE,1535-1537.

Repasky, K. S., J. A. Shaw, J. L. Carlsten, M. D. Obland, L. S. Meng, and D. S. Hoffman, 2004: Diode laser transmitter for water vapor dial measurements. Proc. IEEE Int. Geoscience and Remote Sensing Symp., Anchorage, AK, IEEE, 1947-1950.

Rothman, L. S., and Coauthors, 2003: THE HITRAN molecular spectroscopic database. J. Quant. Spectrosc. Radiat. Transfer, 82, 5-44.

Switzer, G. W., 2007: Semiconductor laser transmitter for water lidar on Mars. Ph.D. dissertation, Montana State University, $193 \mathrm{pp}$.

Trenberth, K. E., and Coauthors, 2007: Observations: Surface and atmospheric climate change. Climate Change 2007: The Physical Science Basis, S. Solomon et al., Eds., Cambridge University Press, 235-336.

Turner, D. D., W. F. Feltz, and R. A. Ferrare, 2000: Continuous water vapor profiles from operational ground-based active and passive remote sensors. Bull. Amer. Meteor. Soc., 81, 1301-1318.

—, R. A. Ferrare, L. A. Heilman Brasseur, W. F. Feltz, and T. P. Tooman, 2002: Automated retrievals of water vapor and aerosol profiles from an operational Raman lidar. J. Atmos. Oceanic Technol., 19, 37-50.

Weckwerth, T. M., V. Wulfmeyer, R. M. Wakimoto, R. M. Hardesty, J. W. Wilson, and R. M. Banta, 1999: NCARNOAA Lower Tropospheric Water Vapor Workshop. Bull. Amer. Meteor. Soc., 80, 2339-2357.

Wulfmeyer, V., 1998: Ground-based differential absorption lidar for water vapor and temperature profiling: Development and specifications of a high performance laser transmitter. Appl. Opt., 37, 3804-3824.

_ tion lidar for water vapor profiling: Assessment of accuracy, resolution, and meteorological applications. Appl. Opt., 37, 3825-3844.

- and C. Walther, 2001: Further performance of ground based and airborne water vapor differential absorption lidar: I. Overview and theory. Appl. Opt., 40, 5304-5320. 